\title{
EIN SÜNDENFALL IN DER ORANGERIE: MÖRIKES ,NACHMÄRZLICHER' MOZART
}

\author{
Ein Attentat oder Wie alles anfing...
}

Am Anfang der Vormärzzeit steht nicht, wie es in der Bibel heißt, das Wort, sondern die Tat, genauer gesagt, ein spektakulärer Terrorakt, ein politisches Attentat mit jahrzehntelangen Auswirkungen. Der 23jährige Student und radikale Burschenschaftler Karl Ludwig Sand ermordet am 23. März 1819 mit seinem Dolch den Dichter August von Kotzebue, in dem er einen russischen Spion und Erzfeind der Burschenschaftsidee vermutet. Was heute in einer sensationsgesättigten Medienwelt allenfalls einen Tag lang eine Karawane von Kamerateams auf den Plan riefe, das löste im Jahre 1819 eine jahrelange Unterdrückungswelle und den kulturellen Kahlschlag aus, der als ein Höhepunkt restaurativer Politik oder kurz als sogenannte Demagogenverfolgung übermittelt ist.

Sands Anschlag ist offensichtlich die Einzeltat eines verwirrten jungen Mannes, aber sie wird zum nicht ganz unwillkommenen Anlass genommen, jetzt mit bislang unbekannter Härte mit der politischen Hatz gegen kritische Geister und querdenkerische Intellektuelle vorzugehen. Diese Politik der Gängelung und Entmündigung manifestiert sich in den umfangreichen Bestimmungen der Karlsbader Beschlüsse, die mit ihrer offensichtlichen Zensurpraxis und massiven Unterdrückungspolitik die Weichen dafür stellen, eine ganze Generation mundtot zu machen. Ihr Maßnahmenkatalog beförderte zwangsläufig eine Atmosphäre der politischen Windstille und erzwang eine Stagnation, während derer viele Autoren sich anpassten, resignierten oder rebellierten, indem sie etwa in die Reiseprosa oder gleich ins benachbarte Ausland flohen, dort ins Exil gingen.

Die zwanziger Jahre des 19. Jahrhunderts sind, so gesehen, eine Zeit der Anpassung und damit eines klebrigen Opportunismus wie fataler Anpassungssucht gewesen. Die Konfiguration dieses verschlafenen und überangepassten Daseins in den damaligen Duodezfürstentümern, das dann als Strömung des Biedermeiers in die 
Literaturgeschichte eingeht, ist besonders treffend in der Figur des Nachtwächters mit Kerzenlicht und Zipfelmütze karikiert worden.

Das immer ein bisschen verstaubte und langweilige Image der Biedermeierzeit war offensichtlich auch durch die signifikante Beflissenheit und ein schwerfällig anmutendes Dichterethos ohne jede Ausstrahlung geprägt. Das Biedermeierliche deckt sich nicht allein schon mit dem Stillhalten und dem Opportunismus eines vor jeder Obrigkeit kuschenden Bürgers und seines vorauseilenden Gehorsams. Biedermeier: das ist in literarhistorischem Verständnis auch die Zeit, die die Krise der Spätromantik ablöst und zugleich schon von schwelender Unzufriedenheit und heftiger Zerrissenheit geprägt ist. Die Dichter in dieser Ära leiden am eigenen Unvermögen, sind aber zugleich auch kaum fähig, eigenes utopisches Potential zu aktivieren und eine korrigierende, neue Sinnstiftung einzuleiten resp. selbst zu leisten.

\section{Melancholie und Serenitas - zwei Seiten einer Medaille}

Als signifikantes Beispiel für diesen fließenden Übergang von der Spätromantik zur Biedermeierzeit gilt Eduard Mörikes Künstlerroman Maler Nolten, eine lang geratene Novelle in zwei Teilen, die er während der zwanziger Jahre schreibt, und die schließlich in Goethes Todesjahr 1832 erscheint (Mörike, 1997) ${ }^{1}$.

Der Handlungsverlauf sei hier nur knapp in Erinnerung gerufen: Der junge Maler Theobald Nolten gewinnt über Förderer Zugang zur Gesellschaft, in der Kunstsinnigkeit, Affären und Liebeleien sich abwechseln, die jedoch kaum jemanden glücklich machen. Täuschung, Intrigen, Briefwirrnisse, Eröffnungen und schließlich eine Reihe von Suiziden sind an der Tagesordnung. Auch ein Theaterstück mit dem Titel Der letzte König von Orplid. Ein phantasmagorisches Zwischenspiel ist neben zahlreichen Gedichten in diesen Roman einmontiert. Das entspricht der Gattungsmischung, die die Romantiker programmatisch machten und so sehr schätzten.

Am Ende der Verwicklungen sind nicht nur alle künstlerischen Aspirationen beendet, sondern auch alle persönlichen Bindungen erweisen sich als brüchig. Bezeichnenderweise sind nahezu alle - gleichgültig ob Intriganten, Freunde, Opfer - tot und vom Profil des Bildungsromans im Stile von Wilhelm Meister - letzterer wird ja immerhin Wundarzt, also Chirurg - weiter denn je entfernt. Mörikes spätromantische Züge aufzeigendes, düsteres Werk vom Maler Nolten spiegelt die schwermütige Zeit des Übergangs, der schicksalhaften Verführung zu Schein und Zerrissenheit - und endet konsequent in der großen ,Nicht-Utopie Tod' (Ernst Bloch).

Bei den zahlreichen lyrischen Einschaltungen im Maler Nolten handelt es sich um Gedichte, die später zuweilen aus dem Roman herausgenommen, z.B. für den

\footnotetext{
${ }^{1}$ Nachfolgend im Text zitiert mit der Sigle MN und der entsprechenden Seitenzahl.
} 
anschaulichen Schulunterricht ausgekoppelt, also gleichsam dem verschlungenen Romanzusammenhang entfremdet wurden. So ist dem Werk unter anderem auch jenes populäre Frühlingsgedicht eingefügt, das zwar allseits als bekannt zitiert wird, aber sicherlich nicht in diesem Kontext zu erwarten war:

Frühling läßt sein blaues Band/ Wieder flattern durch die Lüfte,/ Süße, wohlbekannte Düfte/ Streifen ahnungsvoll das Land;/ Veilchen träumen schon,/ Wollen balde kommen;/ Horch von fern ein leiser Harfenton! - - Frühling, ja du bist's!/ Frühling, ja du bist's!/ Dich hab ich vernommen! (MN, S. 200)

Gleiches gilt für ein anderes im Roman ansässiges, berühmtes Gedichte Mörikes; es greift die gespensterhafte Sage vom „Feuerreiter“ (MN, S. 31 f.) auf. Wie Heinrich von Kleist oder E. T. A. Hoffmann hat sich auch Eduard Mörike - auch darin noch deutlich spätromantisch geprägt - für das Lugubre, das Abgründige, Unbewusste, Unheimliche, kurz: für das, was mit der Ratio und der aufgeklärten Verstandesleistung nicht zu fassen war, interessiert. Hier werden Geheimnisse inszeniert, allerdings ohne den letzten Schleier lüften zu wollen, wozu eben auch jener „Feuerreiter"-Mystizismus gehört. Und beide, Hoffmann wie Kleist, beschäftigen sich außerdem, wie auch Mörike, mit der Musik und Person Mozarts: Hoffmann thematisiert ihn z. B in der Erzählung Don Juan. Eine fabelhafte Begebenheit, die sich mit einem reisenden Enthusiasten zugetragen; zu Kleists Verhältnis zu ihm siehe Kreuzer (1989/90) und Siebert (2005).

Die spätromantischen Anklänge sind aber vielfach klischeehafte Staffage, mehr als auf sie kommt es Mörike auf die Erkundung der Seele, deren ,wahlverwandtschaftlicher` Ähnlichkeiten und Gebundenheiten an. Nahezu alle Personen des Romans sind psychischen Gefährdungen ausgesetzt; sie sind allesamt Melancholiker, stets zur Annahme eines düsteren Fatums geneigt. Doch die Abgründe ihres Schicksals sind keine äußerlichen und tun sich vielmehr in ihnen selbst auf. Insofern sind schon beim frühen Mörike pathologische, psychiatrische Phänomene berührt, oder wie Helmut Koopmann befunden hat:

\footnotetext{
Wahnsinn ist in diesem Roman, kurz gesagt, eine soziale Krankheit, wobei Ursache und Folge nicht eindeutig voneinander zu trennen sind, sondern gleichsam ein zwanghaftes Netz darstellen, aus dem kein Entrinnen möglich ist: soziale Verhältnisse führen zum Wahnsinn, und der Wahnsinn wiederum steigert die soziale Unfähigkeit des Menschen. (Koopmann, 1997, S. 1021)
}

Mörike schreibt demnach mit seinem Maler Nolten einen psychologischen Roman, in dem es nicht so sehr um das Phänomen des Wahnsinns selbst, wohl aber um die Möglichkeiten einer Literarisierung von Wahnsinn geht. Das hebt den Wert dieses Romans über den Illustrationsstatus eines klinischen Zeitdokuments der Biedermeier-Epoche hinaus, und es hat ihn als Beispiel für eine sprachliche Bewältigung innerer Zerrissenheit für die Moderne interessant gemacht. 


\section{Von Jungdeutschen und Kosmopoliten}

Ab Mitte des Jahres 1830 scheint endlich, kulturpolitisch gesprochen, die langersehnte Wende zum Aufbruch wieder in Sicht, und wieder kommt das Heil aus Frankreich, wo erneut, eine Veränderung herbeizuführen versucht wird. Signifikant ist der Enthusiasmus, der jetzt auch viele Schriftsteller über die deutschen Länder hinaus erfasst. Der Spötter Heine hat in seiner ungestümen Heftigkeit in seinem Urteil noch das ganze Maß vorheriger Niedergedrücktheit plastisch werden lassen:

\footnotetext{
Ist es wirklich wahr, daß das stille Traumland in lebendige Bewegung geraten? Wer hätte das vor dem Julius 1830 denken können! Goethe mit seinem Eiapopeia, die Pietisten mit ihrem langweiligen Gebetbücherton, die Mystiker mit ihrem Magnetismus, hatten Deutschland völlig eingeschläfert, und weit und breit, regungslos, lag alles und schlief. Aber nur die Leiber waren schlafgebunden; die Seelen, die darin eingekerkert, behielten ein sonderbares Bewußtsein. Der Schreiber dieser Blätter wandelte damals, als junger Mensch, durch die deutschen Lande und betrachtete die schlafenden Menschen; ich sah den Schmerz auf ihren Gesichtern, ich studierte ihre Physiognomien, ich legte ihnen die Hand aufs Herz und sie fingen an nachtwandlerhaft im Schlafe zu sprechen, seltsam abgebrochene Reden, ihre geheimsten Gedanken enthüllend. Die Wächter des Volks, ihre goldenen Nachtmützen tief über die Ohren gezogen, und tief eingehüllt in Schlafröcken von Hermelin, saßen auf roten Polsterstühlen, und schliefen ebenfalls, und schnarchten sogar. Wie ich so dahinwanderte, mit Ränzel und Stock, sprach ich oder sang ich laut vor mich hin, was ich den schlafenden Menschen auf den Gesichtern erspäht oder aus den seufzenden Herzen erlauscht hatte; - es war sehr still um mich her, und ich hörte nichts als das Echo meiner eigenen Worte. Seitdem, geweckt von den Kanonen der großen Woche, ist Deutschland erwacht, und jeder, der bisher geschwiegen, will das Versäumte schnell wieder einholen, und das ist ein redseliger Lärm, und ein Gepolter, und dabei wird Tabak geraucht und aus den dunklen Dampfwolken droht ein schreckliches Gewitter. Das ist wie ein aufgeregtes Meer, und auf den hervorragenden Klippen stehen die Wortführer; die einen blasen mit vollen Backen in die Wellen hinein, und sie meinen, sie hätten diesen Sturm erregt und je mehr sie bliesen desto wütender heule die Windsbraut; die anderen sind ängstlich, sie hören die Staatsschiffe krachen, sie betrachten mit Schrecken das wilde Gewoge, und da sie aus ihren Schulbüchern wissen, daß man mit Öl das Meer besänftigen könne, so gießen sie ihre Studierlämpchen in die empörte Menschenflut, oder prosaisch zu sprechen, sie schreiben ein versöhnendes Broschürchen, und wundern sich, wenn das Mittel nicht hilft und seufzen: ,Oleum perdidi!' Es ist leicht vorauszusehen, daß die Idee einer Republik, wie sie jetzt viele deutsche Geister erfaßt, keineswegs eine vorübergehende Grille ist. (Heine, 1997, S. 209)
}

Zweifellos: unter dem Signet des Republikanismus sammeln sich fortan diejenigen, die die Literaturgeschichte mit dem Begriff vom „Jungen Deutschland“ fassen wird. Der junge Heinrich Laube geht noch einen Schritt weiter und schreibt in den Jahren 1833-37 eine umfängliche Romantrilogie mit dem programmatischen Titel Das junge Europa. Am Beispiel eines Briefwechsels zwischen fünf jungen Europäern, die unterschiedlichen Temperaments, unterschiedlicher politischer Auffassung, unterschiedlicher Lebensphilosophie sind, werden die stimulierenden Wirkungen der Juli-Revolution - Aufbruch, Begeisterung, politisches Engagement im Freiheitskampf, schließlich Ernüchterung und Desillusionierung - aufgezeigt und reflektiert (Thielking, 2000, S. 48 ff.). 
Nicht allzu lange hielt der Enthusiasmus der Jungdeutschen bzw. Jungeuropäer vor, denn bald schon erwiesen sich die realen Zustände und ihre Folgen als marode und ließen sich schon gar nicht auf Freiheitsträume übertragen. Statt Freiheitssonne und republikanischer Veränderung wurden auch weiterhin die kritischen Geister von der Zensur im Zaum gehalten, polizeiliche Maßnahmen lähmten das literarische Feld des Widerstands, eine kritische Einmischung erfolgte allenfalls aus dem Exil heraus.

Das bedeutendste Zeugnis einer solch radikalen Anklage dürfte Heinrich Heines fingierte Reiseprosa in Deutschland, ein Wintermärchen von 1844 sein, in der Heine das reaktionäre Deutschland verspottete und zugleich sich zum populären Sänger einer neuen, sozial und materiell gerechteren und ästhetisch genussreicheren Zeit aufschwang. In der Tat dürfte sie für die politische Reiseliteratur und auch das Reisefeuilleton formgebend und vorbildlich gewirkt haben.

Doch die sozialen Aufstände und Hungerrevolten der Jahre 1844/45, wie sie Heine in seinem Lied der Schlesischen Weber prägnant fasste, sind nicht der Auslöser der Revolutionen von 1848/49. Sie sind eine europäische Protestbewegung mit vielschichtigen, zum Teil divergierenden Interessenslagen, bei der das liberale Bürgertum konstitutionelle Mitspracherechte gegenüber monarchischer Willkür fordert. Das ökonomisch erstarkende Bürgertum verlangt nach der angemessenen Beteiligung an der politischen Macht. Zugleich artikulieren sich auch erste frühproletarische Ansprüche nach sozialer Anerkennung und Gerechtigkeit. Die Märzrevolution von 1848 - nach ihr benannt, zerfällt diese Zeit in den ,Vormärz' und den ,Nach-

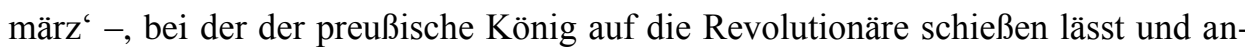
schließend gezwungen wird, den vor seinem Schloss aufgebahrten Opfern und Toten seine Reverenz zu erweisen, ist das eigentliche Kernstück eines politischen Ermächtigungsvorgangs. In einer zweiten Phase wird der bürgerliche Wunsch nach Freiheit und Verfassung zuungunsten einer nun oktroyierten Verfassung zurückgeworfen, die Preußendeutschland zur konstitutionellen Monarchie eines langen ,Nachmärzes ‘ zurückkehren lässt, der in die Geschäftigkeit des Frührealismus übergeht.

\section{„Die neunte Muse soll nicht zu kurz kommen“"2: Mörikes (nach)märzlicher Mozart}

So ganz anders als Heines scharfzüngige sozialkritische Anklagen und seine kühne Zukunftspolitik für die Künste, die mitunter mit Pauken und Trompeten lautstark für radikale Veränderung einsteht, zeigt sich Mörikes Reaktion auf die Revolutionen von 1848/49. Während der revolutionären Unruhen in den großen europäischen Metropolen, darunter auch in Wien, hat er die kleine Erzählung Mozart auf

\footnotetext{
${ }^{2}$ Aus: Mörikes Mozart auf der Reise nach Prag, S. 583, nachfolgend im Text mit der Sigle MRP und der entsprechenden Seitenzahl zitiert.
} 
der Reise nach Prag begonnen, die erst 1855 im Morgenblatt für gebildete Leser erscheint und als Buchausgabe auf das Jahr 1856 - Mozarts einhundertsten Geburtstag - vordatiert wurde.

Mörikes im Nachmärz angesiedelte Mozart-Novelle umkreist das Themenspektrum Heiterkeit und Schwermut (Fingerhut, 2011, S. 93-109); entsprechend scheint sie nur auf den ersten Blick keinen Zusammenhang mit der aktuellen politischen Situation zu haben. Worum geht es in ihr? Geschildert werden fiktive vierundzwanzig Stunden im Leben des berühmten Komponisten Wolfgang Amadeus Mozart, der sich auf der Reise von Wien nach Prag befindet (umfassend zur Beziehung Mörikes zu Mozart: Meyer, 1985, S. 29-40). Es gibt andeutungsweise einen realgeschichtlichen Hintergrund: Eine derartige Reise nach Prag hat Mozart zur geplanten Uraufführung seiner Oper Don Giovanni (1789) tatsächlich unternommen.

In Mörikes Erzählung macht Mozart bei seiner Anreise eine mittägliche Rast und begibt sich zum Spaziergang in den nahegelegenen gräflichen Gartenpark der Schinzbergs, um sich nach beschwerlicher Reise die Beine ein wenig zu vertreten. Dort fasziniert und fesselt den Musiker und Komponisten schon bald das heitere Geplätscher eines Springbrunnens und mehr noch der Anblick eines prunkenden Pomeranzenbaumes (zur schon sehr viel früher einsetzenden Beliebtheit des Pomeranzenschmucks: Baumgartner, 2005; vgl. auch Gröschel, 2005), dessen kostbare Früchte eine spontane Erinnerung in ihm wachrufen. Die Erinnerung, die ein solcher locus aтоеnus in ihm wachruft, sowie die plötzliche Eingebung einer neuerlichen künstlerischen Inspiration, mischen sich in einem dichten, gleichsam sensualistisch aufgeladenen Augenblick zu einem Initiationsakt musikalischer Neuschöpfung. Dabei kommt es zu Mozarts unbewusster handgreiflicher Reaktion und damit zu jenem „Unstern in der Laube“ (MRP, S. 588), wie es später scherzhaft heißt, und damit zum folgenreichen Übergriff in der Orangerie, die ein Gärtner, der zur Wache und als „Gartenpolizei“ (MRP, S. 588) bestellt, prompt als Diebstahl auffasst. Die Schlüsselstelle der Erzählung leitet das sinnig ein:

Das Ohr behaglich dem Geplätscher des Wassers hingegeben, das Aug auf einen Pomeranzenbaum von mittlerer Größe geheftet, der außerhalb der Reihe, einzeln, ganz dicht an seiner Seite auf dem Boden stand und voll der schönsten Früchte hing, ward unser Freund durch diese Anschauung des Südens alsbald auf eine liebliche Erinnerung aus seiner Knabenzeit geführt. Nachdenklich lächelnd reicht er hinüber nach der nächsten Frucht, als wie um ihre herrliche Ründe, ihre saftige Kühle in hohler Hand zu fühlen. Ganz im Zusammenhang mit jener Jugendszene aber, die wieder vor ihm aufgetaucht, stand eine längst verwischte musikalische Reminiszenz, auf deren unbestimmter Spur er sich ein Weilchen träumerisch erging. (MRP, S. 579)

Der freundliche und doch distanzierte Duktus des auktorialen Erzählers kommentiert den „Wandler im Park“ (zum Typus siehe: Gröning, 2003) und den genießenden Akteur durchaus einnehmend (,unser Freund“) und zeigt die immense Wirkung auf, die das plötzliche Handanlegen auf den überraschenden Zugreifer zeitigt. Der Tempuswechsel ins Präsens markiert und hält gewissermaßen überdies die 
besonders traumverloren wirkende somnambule Wendung, die nun erinnernd mit ihm vor sich geht:

Jetzt glänzen seine Blicke, sie irren da und dort umher, er ist von einem Gedanken ergriffen, den er sogleich eifrig verfolgt. Zerstreut hat er zum zweitenmal die Pomeranze angefaßt, sie geht vom Zweige los und bleibt ihm in der Hand. (MRP, S. 579)

Was nun folgt, ist die Darstellung einer Ablenkung, die das künstlerische Genie erfährt und die erneut durch Wendungen ins Paradoxe an Dichte des Eindrucks, an Glaubwürdigkeit und Authentizität gewinnt. Die Synästhesie der optischen, haptischen, aromatischen und olfaktorischen Benommenheit erzeugt und stützt zudem den vorbewussten Zustand einer Erregung des Künstlers, schafft die schwebende Stimmung der Vagheit für einen symbolischen und quasischöpferischen Initiationsakt in Schnitt und Zusammenfügung der beiden Fruchthälften:

\footnotetext{
Er sieht und sieht es nicht; ja so weit geht die künstlerische Geistabwesenheit, daß er, die duftige Frucht beständig unter der Nase hin und her wirbelnd und bald den Anfang, bald die Mitte einer Weise unhörbar zwischen den Lippen bewegend, zuletzt instinktmäßig ein emailliertes Etui aus der Seitentasche des Rocks hervorbringt, ein kleines Messer mit silbernem Heft daraus nimmt und die gelbe kugelige Masse von oben nach unten langsam durchschneidet. Es mochte ihn dabei entfernt ein dunkles Durstgefühl geleitet haben, jedoch begnügten sich die angeregten Sinne mit Einatmung des köstlichen Geruchs. Er starrt minutenlang die beiden innern Flächen an, fügt sie sachte wieder zusammen, ganz sachte, trennt und vereinigt sie wieder. (MRP, S. 579 f.)
}

Die Quellen, aus denen diese Aktion schöpft, die Beweggründe sind vage und nur unbestimmt spekulierend angedeutet (,Es mochte ihn ... geleitet haben“). Nirgends wird Gewissheit erzeugt und durch ein Modalverb bestärkt. Hier erfolgen signifikante Ersatzhandlungen; hier agiert einer spontan und ungeschützt, dessen Sinne vielfach (über)angeregt werden, und der folglich, selbstvergessen und instinktgeleitet, wie in Trance vorzugehen scheint, bis ihn die Wirklichkeit des Parkgartens ernüchternd einholt, und seine seltsame Handlungsweise ihm ins helle Bewusstsein hebt und entlarvt.

\begin{abstract}
Da hört er Tritte in der Nähe, er erschrickt, und das Bewußtsein, wo er ist, was er getan, stellt sich urplötzlich bei ihm ein. Schon im Begriff, die Pomeranze zu verbergen, hält er doch gleich damit inne, sei es aus Stolz, sei's weil es zu spät dazu war. Ein großer breitschulteriger Mann in Livree, der Gärtner des Hauses, stand vor ihm. Derselbe hatte wohl die letzte verdächtige Bewegung noch gesehen und schwieg betroffen einige Sekunden. Mozart, gleichfalls sprachlos, auf seinem Sitz wie angenagelt, schaute ihm halb lachend, unter sichtbarem Erröten, doch gewissermaßen keck und groß mit seinen blauen Augen ins Gesicht; dann setzte er - für einen Dritten wäre es höchst komisch anzusehen gewesen - die scheinbar unverletzte Pomeranze mit einer Art von trotzig couragiertem Nachdruck in die Mitte des Tisches. (MRP, S. 580)
\end{abstract}

Der Gärtner als Aufseher stellt ihn zur Rede, glaubt er den Frevler doch auf frischer Tat ertappt und des Fruchtdiebstahls überführt zu haben. Der ins Visier der Unbotmäßigkeit Geratene reagiert empört: 
„Was?“ rief Mozart, „Zugreifen? Zum Teufel, glaubt Er denn, ich wollte stehlen und das Ding da fressen?““ „Mein Herr, ich glaube was ich sehe. Diese Früchte sind gezählt, ich bin dafür verantwortlich. Der Baum ist vom Herrn Grafen zu einem Fest bestimmt, soeben soll er weggebracht werden. Ich lasse Sie nicht fort, ehbevor ich die Sache gemeldet und Sie mir selbst bezeugten, wie das da zugegangen ist.“(MRP, S. 580)

Die Lage dessen, der sich zu so etwas auf den ersten Blick Unerklärlichem hat hinreißen lassen, erscheint prekär, der Ausgang des Vergehens so ungewiss, dass der Täter dem Gärtner nicht die Überbringung der Botschaft allein überlassen will, also muss rasch ein verständiges Schreiben her, das, hingekritzelt, Mozart zur Erklärung und Abbitte zwingt:

Zwei Gartenknechte kamen nun wirklich herbei, luden den Baum auf eine Bahre und trugen ihn hinweg. Inzwischen hatte unser Meister seine Brieftasche gezogen, ein weißes Blatt herausgenommen, und während daß der Gärtner nicht von der Stelle wich, mit Bleistift angefangen zu schreiben: „Gnädigste Frau! Hier sitze ich Unseliger in Ihrem Paradiese, wie weiland Adam, nachdem er den Apfel gekostet. Das Unglück ist geschehen, und ich kann nicht einmal die Schuld auf eine gute Eva schieben, die eben jetzt von Grazien und Amoretten eines Himmelbetts umgaukelt, im Gasthof sich des unschuldigsten Schlafes erfreut. Befehlen Sie und ich stehe persönlich Ihro Gnaden Rede über meinen mir selbst unfaßlichen Frevel. Mit aufrichtiger Beschämung Hochdero untertänigster Diener W. A. Mozart, auf dem Wege nach Prag.“ (MRP, S. 581)

Die delikate Affäre löst sich schnell und unkompliziert, ja dezidiert generös und weltmännisch, auf. Im Schloss erweist sich der Komponist dem gräflichen Paar als bekannt. Als schönstes Geschenk zu ihrer Verlobung erhält Eugenie, die Tochter des Grafen Schinzberg, eine exklusive musikalische Vorabdarbietung der Don Giovan$n i$-Komposition. Erst am nächsten Tag setzt das Ehepaar Mozart, ungewöhnlich reich mit einer prächtigen Kutsche beschenkt, die Fahrt nach Prag fort. Als überaus heiteres und geselliges Musikergenie bleibt der scheidende Mozart den Gastgebern in bester Erinnerung, die einzig überschattet von der Ahnung seines frühen Todes ist.

\section{Nota bene}

Doch was könnte ungeachtet dieses idyllenhaften Ausklangs die eben zitierte Passage, Mozarts Fauxpas in der Orangerie des Adels, doch untergründig mit nachmärzlicher, noch immer revolutionärer Politik zu tun haben? Die zugrunde gelegte Handlung spielt im Herbst 1787, also kurz vor dem Niedergang des ancien régime in der anderen, vorgängigen revolutionären Situation von 1789, die selbstverständlich den großen Erfahrungsbezugspunkt für die aktuellen Ereignisse 1848 bildete. Denn womit vergleicht man ungeheuerliche Geschehnisse? In erster Linie wohl mit dem kulturell Vorrätigen an Vergangenem.

Die Schinzbergs, deren repräsentatives Prachtstück Mozart in dem ererbten Pomeranzenbaum beschädigt, sind aufgeklärte, kultivierte und freigiebige Adelige. 
Mozart vergreift sich - unbewusst, weil gleichsam in künstlerischer Trance von der Komposition seines Don Giovanni abgelenkt - an der exotischen Frucht, die, an einem 25jährigen Bäumchen gediehen, im Text selbst „als lebendes Symbol der feingeistigen Reize eines beinahe vergötterten Zeitalters" (MRP, S. 596) bezeichnet wird. Von hier aus lässt sich die Handlung des Komponisten, dessen ganz unkonventioneller Habitus ohne Prunk, abgeschabt und mit einem nachlässig getragenen Zopf dargestellt wird, sozial und künstlerisch parallelisieren. In diesem Deutungshorizont nimmt der bürgerliche Künstler gleichsam symbolisch den von der Revolution herbeigeführten Übergriff auf die Besitztümer der Adeligen vorweg. Mörikes Novelle beschreibt am Ende Mozarts Innovation, und es fällt die latente Revolutionsmetaphorik daran auf: Der Schlusschoral

erklang durch die Totenstille des Zimmers. Wie von entlegenen Sternenkreisen fallen die Töne aus silbernen Posaunen, eiskalt, Mark und Seele durchschneidend, herunter durch die blaue Nacht. (....) Nachdem diese dröhnenden Klänge bis auf die letzte Schwingung in der Luft verhallt waren, fuhr Mozart fort: „Jetzt gab es für mich begreiflicherweise kein Aufhören mehr. Wenn erst das Eis einmal an einer Uferstelle bricht, gleich kracht der ganze See und klingt bis in die entferntesten Winkel hinunter.“(MRP, S. 616)

Zugleich aber beschreibt Mörikes erzählerische Analogie im Grunde nichts anderes als die augenblickliche autobiographische Situation. Überall ringsum in allen europäischen Metropolen und fast allen Landschaften, und darunter ganz besonders im Südwestdeutschen, wo Mörike lebte und arbeitete, war nach einer Zeit politischer Totenstille das Eis gebrochen, der Wunsch nach Freiheit in vielfältiger Weise, auch jubilierend und in Musik festgehalten, unsterblich zum Ausdruck gebracht worden.

\section{Bibliographie}

Baumgartner, T. (2005)., Welsche Pamben, Feigenheiser und Pumerantschenstuben“. Streiflichter auf die Entwicklung der Orangeriekultur im Wiener, nieder- und oberösterreichischen, Salzburger und Tiroler Raum von den Anfängen bis 1683. In Ders. (Hrsg.), Ein Hauch von Gold. Pomeranzen und Gartenkunst im Passauer Land (S. 131-158). Passau: Schnell + Steiner.

Fingerhut, K. (2011). Amadeus o la potenza della musica. Mozart nella letteratura romantica tedesca (tradotto dal tedesco da Fabio Olivetti, Trento). In S. Capellari (Ed.), Il personaggio di Mozart nella letteratura d'invenzione. Atti del Convegno Verona Academia Filarmonica 4.-5. dicembre 2007 (S. 93-109). Verona: Qui Edit.

Gröning, G. (2003). Der Wandler im Park. Ein prasialogischer Beitrag zur Gartenkultur des 19. Jahrhunderts mit besonderer Berücksichtigung der ,Neuen Gartenbaukunst ${ }^{\star}$ von Friedrich Gotthelf Baumgärtner. In G. Bayerl \& T. Meyer (Hrsg.), Die Veränderung der Kulturlandschaft. Nutzungen - Sichtweisen - Planungen (S. 103-129). Münster: Waxmann.

Gröschel, C. (2005). Großer Herren Vergnügen - Orangeriepflanzen als Motiv der höfischen Kunst. In T. Baumgartner (Hrsg.), Ein Hauch von Gold. Pomeranzen und Gartenkunst im Passauer Land (S. 131-158). Passau: Schnell + Steiner. 
Heine, H. (1997). Französische Zustände. In Ders., Sämtliche Schriften, Bd. 3. Hrsg. v K. Briegleb. (S. 89-279). München: dtv.

Koopmann, H. (1997). Nachwort. In E. Mörike, Sämtliche Werke. Bd.1 (6. Aufl.), (S. 1001-1026) Darmstadt: Wissenschaftliche Buchgesellschaft.

Kreuzer, H.-J. (1989/1990). Hat Kleist Mozart gesehen? Mozart-Jahrbuch [o. Nr.], 141-148.

Meyer, H. (1985). Das Fortleben Mozarts bei Mörike und seinen Freunden. Acta Mozartiana, 32, H. 1, $29-40$.

Mörike, E. (1997). Maler Nolten. In Ders., Sämtliche Werke Bd. I (6. Aufl.), (S. 5-383), Darmstadt: Wissenschaftliche Buchgesellschaft (Sigle MN).

Mörike, E. (1997). Mozart auf der Reise nach Prag. In Ders., Sämtliche Werke, Bd. I (6. Aufl.), (S. 566-622). Darmstadt: Wissenschaftliche Buchgesellschaft (Sigle MRP).

Siebert, E. (2005). Noch einmal Kleist und Mozart. Heilbronner Kleist-Blätter 17, 137-145.

Thielking, S. (2000). Weltbürgertum. Kosmopolitische Ideen in Literatur und politischer Publizistik seit dem achtzehnten Jahrhundert. München: Fink. 